\title{
High Performance Liquid Chromatography
}

National Cancer Institute

\section{Source}

National Cancer Institute. High Performance Liquid Chromatography. NCI Thesaurus. Code C16434.

Frequently referred to simply as HPLC, this form of column chromatography is used frequently in biochemistry. The analyte is forced through a column by liquid at high pressure, which decreases the time the separated components remain on the stationary phase. Less time on the column then translates to narrower peaks in the resulting chromatogram and thence to better selectivity and sensitivity. 